\title{
HIGH-RESOLUTION B-PHOTOMETRY OF THE MILKY WAY AND RESULTS ON GALACTIC STRUCTURE
}

\author{
S. Kimeswenger, ${ }^{1,2}$ W. Schlosser, ${ }^{1}$ K. J. Seidensticker, ${ }^{1}$ B. Hoffmann, ${ }^{1}$ \\ and Th. Schmidt-Kaler ${ }^{1}$ \\ ${ }^{1}$ Astronomical Institute \\ Ruhr-Universität \\ Bochum Postfach 102148 \\ D-4630 Bochum FRG \\ ${ }^{2}$ Institute of Astronomy \\ University of Innsbruck \\ Technikerstraße 25 \\ A-6020 Innsbruck Austria
}

\section{INTRODUCTION}

During the last two decades, many attempts were made to determine the global parameters of the Galaxy and to compare the Galaxy to other galaxies (Schmidt-Kaler and Schlosser 1973; de Vaucouleurs and Pence 1978; Gilmore 1984; van der Kruit 1986). While most of these investigations are based on star counts, a detailed overall study by surface photometry, because of the lack of homogeneous high-resolution data, is rare. The last attempt by van der Kruit (1986), based on Pioneer 10 data, suffered from low resolution. The great number of individual structures at low and even intermediate latitudes could not be recognized. Our work (Bband, Hoffmann et al. 1989, this volume; V-band, Schlosser, Schmidt-Kaler, and Schneider 1989; U-Band and R-band photometry, in preparation) provides this homogeneous highresolution data.

\section{DATA AND METHODS}

The map in Figure 1 is based on exposures with the Bochum super-wide-angle camera. Reduction was done by well-developed standard procedures (Schmidt-Kaler et al. 1983; Hoffmann et al. 1989, this volume). We combined seven plates to a single-surface photometry with a resolution of $0.25 \times 0.25$. Figure 2 shows the combination of $\mathrm{V}$ (Hoffmann et al. 1989, this volume) and U (Schlosser, Schmidt-Kaler, and Schneider 1989) photometries of the same resolution.

\section{DISCUSSION}

Figure 1 shows prominent nearby dark clouds (e.g., "Coalsack," Norma clouds and Ophiuchus region) and the prominent star-forming regions in the Carina Arm (-I). The outer isophotes are dominated by the global parameters of the bulge, the "main body" (Schmidt-Kaler and Schlosser 1973), and the disc. The map in Figure 2 is not dominated by local structures, but by the global properties of the Galaxy. Even the northern part of the bulge-almost totally obscured in the intensity map by nearby dark clouds-comes out clearly. The disc colors show good symmetry with respect to the galactic plane up to the region on the right-hand side where the blue Gum Nebula in Vela-Puppis comes up. Note the strong extinction band along $b=0^{\circ}$. The band starts at $l=284^{\circ}$, the tangential point of the Carina Arm (-I). We tried to estimate the distance of this central dust lane, which is getting more prominent with increasing longitude, by five different methods. This leads to a distance of about $580 \pm 100 \mathrm{pc}$. 


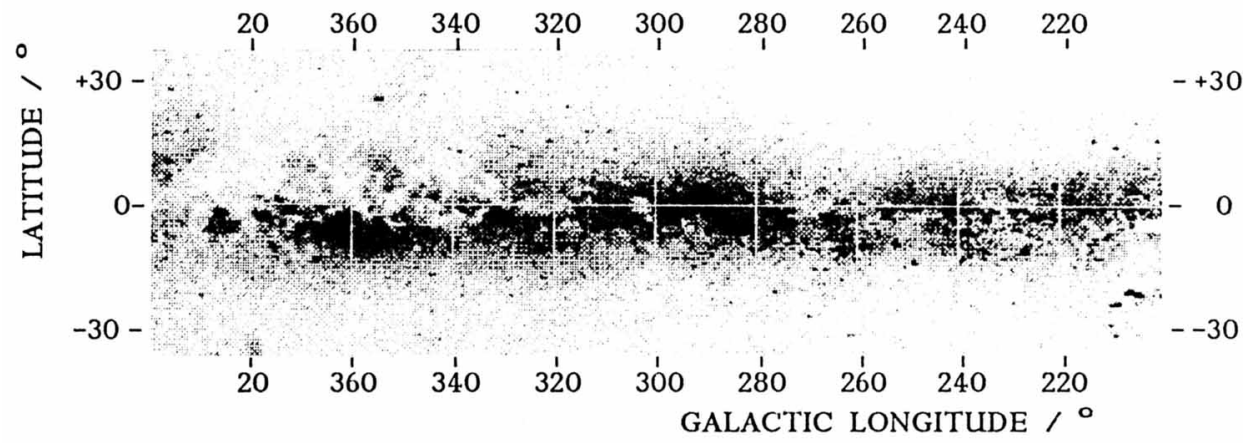

Figure 1. Surface brightness of the Milky Way in B-band. Stars brighter than $m_{B}=7.5^{\mathrm{m}}$ were removed. Note the prominent local dust clouds and the bright Carina star-forming regions.

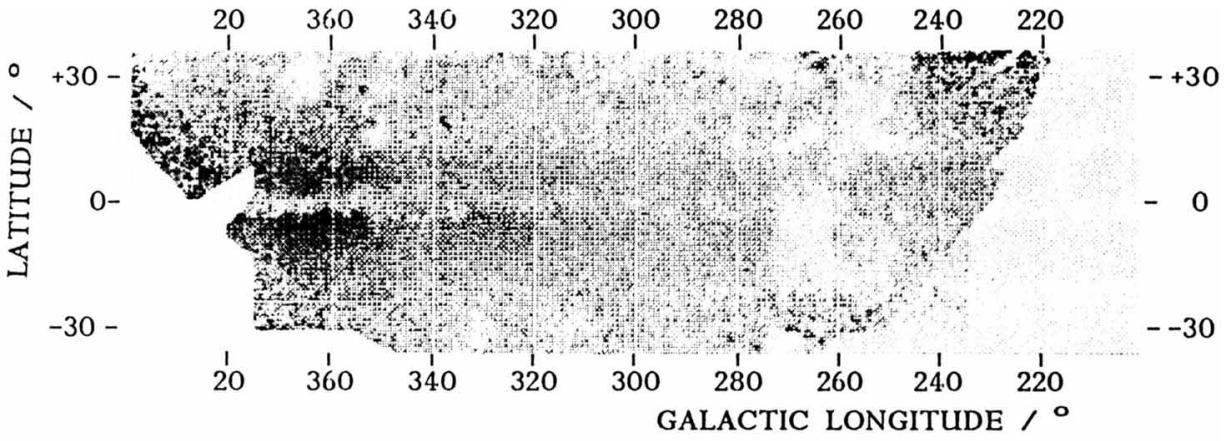

Figure 2. U-V color map of the Milky Way (dark = red; light = blue). The map is not dominated by local features, but by the global parameters of the galaxy.

\section{REFERENCES}

de Vaucouleurs, G. and Pence, W. D. 1978, Astron. J., 83, 1163.

Gilmore, G. 1984, MNRAS, 207, 223.

Hoffmann, B. et al. 1989, in Proceedings of IAU Symposium \#139, Galactic and Extragalactic Background Radiation, eds. S. Bowyer and C. Leinert, Kluwer Academic Publisher, Dordrecht, The Netherlands.

Schlosser, W., Schmidt-Kaler, Th., and Schneider, H. 1989, Astron. Astrophys., in preparation.

Schmidt-Kaler, Th. and Schlosser, W. 1973, Astron. Astrophys., 29, 409.

Schmidt-Kaler, Th. et al. 1983, Astron. Astrophys. Suppl., Ser. 51, 1.

van der Kruit, P. C. 1986, Astron. Astrophys., 157, 230. 